\title{
Efecto de la cantidad de diésel en la calidad de las tejas
}

\section{Effect of diesel amount on the quality of the tiles}

\author{
Lilibeth Madrid ${ }^{1}$, José Peralta ${ }^{l}$ Yasury Huertas ${ }^{l}$, Cristian Peralta ${ }^{1}$, Bolivar Bernal ${ }^{2 *}$ \\ ${ }^{1}$ Licenciatura en Ingeniería Industrial-Centro Regional de Azuero-Universidad Tecnológica de Panamá, ${ }^{2}$ Docente de pregrado- \\ Facultad de Ingeniería Industrial-Centro Regional de Azuero-Universidad Tecnológica de Panamá
}

\begin{abstract}
Resumen En este artículo se exhiben los resultados experimentales obtenidos al someter el proceso de fabricación de tejas de arcillas, a la regulación en la cantidad de diésel empleado en el proceso para su deslizamiento y que tiene influencia en la calidad del producto final obtenido. Para lograr el propósito, se aplicó un diseño de experimento en la fábrica de Arcillas S.A. que consistió en la toma de muestras de distintos días de producción bajo dos parámetros: (1) diésel no regulado y (2) diésel regulado, colocando una válvula para controlar la cantidad de diésel empleado en la fabricación de las tejas. Posteriormente, se contrastaron las hipótesis nula y alterna, aplicando la herramienta estadística de análisis de varianza a los datos resultantes del experimento, permitiendo concluir que existe evidencia significativa para confirmar que la cantidad de diésel aplicado influye en la calidad de la teja que se fabrica en un nivel de confianza del $95 \%$.
\end{abstract}

Palabras clave Arcilla, secado, horneado, defectos estructurales, análisis de varianza.

\begin{abstract}
In this article the experimental results obtained by submitting the clay tile manufacturing process to the regulation of the quantity of diesel used in the process for its sliding and which influences the quality of the final product obtained are shown. To achieve the purpose, an experiment design was applied in the "Clay Factory S.A.". which consisted of the sampling of different days of production under two parameters: 1) unregulated diesel and (2) regulated diesel, placing a valve to control the amount of diesel used in the manufacture of the tiles. Subsequently, the null and alternate hypothesis were tested, applying the statistical tool of analysis of variance to the data resulting from the experiment, allowing to conclude that there is significant evidence to confirm that the amount of diesel applied influences the quality of the tile that is manufactured in a level of confidence of $95 \%$.
\end{abstract}

Keywords: Clay, drying, baking, structural defects, variance analysis.

Corresponding authors: bolivar.bernal@utp.ac.pa

\section{Introducción}

La calidad es un factor clave para el éxito de los negocios, el crecimiento y la obtención de una posición competitiva [1]. El manejo deficiente de los procesos puede ejercer un efecto poderoso en el desempeño de la empresa, casi siempre representa del 15 al $20 \%$ de los productos defectuosos [2].

La fábrica de Arcillas S.A. está ubicada en Chitré, provincia de Herrera, y se dedica a la producción y comercialización de productos derivados de la arcilla entre ellos, diferentes tipos de bloques, tejas, chapas, baldosas, ladrillos y otros accesorios utilizados para techos.

En la actualidad, la empresa presenta inconformidad con la calidad de su producto clave las tejas tipo S (modelos: imperial, castilla y catalana). En esa dirección, se ha realizado una evaluación de las operaciones que forman parte del proceso de fabricación: molienda, extrusión, corte, secado y horneado. De esta manera se determinó como el principal factor de afectación la cantidad de diésel que se añade a la pieza para facilitar su deslizamiento de la extrusora al proceso corte.
La presente investigación busca comprobar o descartar, mediante un diseño experimental, si la cantidad de diésel aplicado afecta la calidad de la teja, identificando el factor influyente (cantidad de diésel), previo a la comparación del porcentaje de producto defectuoso antes y después de regular la cantidad de diésel. Es de importancia señalar que para la realización del estudio se conservaron las mismas condiciones de secado, ciclo de cocimiento y temperatura del horno.

\section{Planteamiento del problema}

Actualmente, un promedio de $30 \%$ de la producción de tejas de la empresa Arcilla S.A., es considerado defectuoso, situación que reduce los niveles de productividad e incrementa los costos de producción ocasionados por la pérdida de materia prima empleada en el proceso.

Se considera que el alto porcentaje de fabricación defectuosa se debe a la cantidad de diésel que se aplica para su deslizamiento entre el proceso de extrusión y corte. En 
virtud de lo expuesto, la investigación busca responder la siguiente interrogante:

¿La cantidad de diésel empleada en el proceso de producción de la teja afecta su calidad?

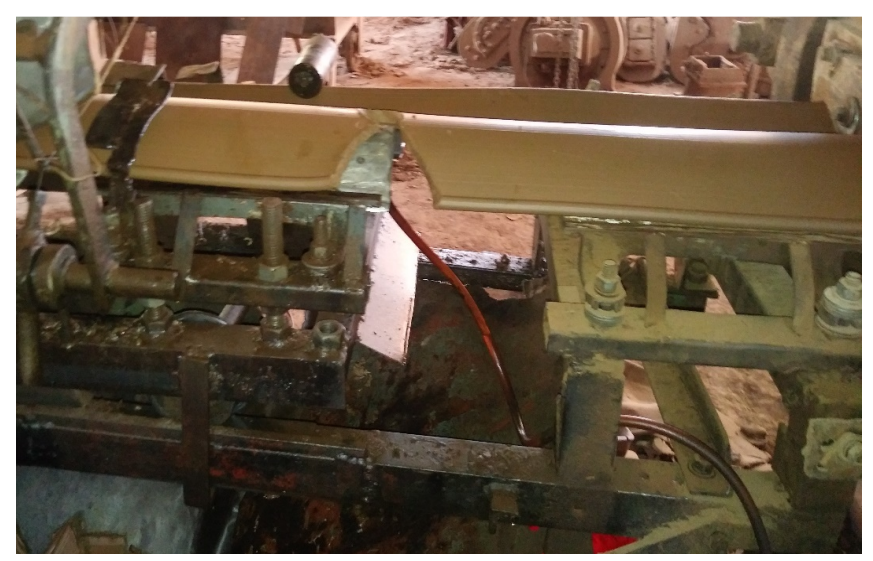

Figura 1. Aplicación de diésel a la teja entre el proceso de extrusión y corte.

\section{Metodología}

\subsection{Elección del diseño experimental}

El diseño experimental consiste en la realización de una o varias pruebas en las cuales se inducen cambios deliberados en las variables de entrada de un proceso o sistema, de manera que sea posible observar e identificar las causas de los cambios en las respuestas de salida [3].

En esta investigación se aplicó un diseño experimental unifactorial al azar, una vez que se identificó, mediante un análisis de todo el proceso de fabricación de la teja, que el factor de mayor afectación era la cantidad de diésel aplicado para facilitar el deslizamiento. La herramienta estadística utilizada para el análisis de los resultados experimentales fue el análisis de varianza, la cual permite separar la variación total en las partes con las que contribuye cada fuente de variación en el experimento, cuyo objetivo es probar la hipótesis de igualdad de los tratamientos con respecto a la media de la correspondiente variable de respuesta [4].

El modelo matemático utilizado para este diseño se muestra en la ecuación (1)

$$
Y_{i j}=\mu+\tau_{i}+\varepsilon_{i j}
$$

Donde:

$Y_{i j}=$ valor observado dentro de cada tratamiento.

$\mu=$ efecto medio verdadero.

$\tau_{i}=$ variable que varía de tratamiento en tratamiento.

$\varepsilon_{i j}=$ error experimental.

\subsection{Variables experimentales}

- Variable de respuesta: Es aquella que permite conocer el efecto o los resultados de cada prueba experimental [4]. En este experimento tendremos como variable de respuesta el porcentaje de tejas defectuosas.

- Factor: Se entiende por factor a aquella variable que afecta la variable de respuesta [4]. En nuestro caso el factor será la cantidad de diésel aplicado.

- Niveles: Son los diferentes valores que se le asignan a cada factor [4]. Para este experimento se definieron dos niveles: cantidad de diésel regulado y cantidad de diésel no regulado.

- Tratamientos: Son la combinación de todos los factores estudiados. En el diseño experimental unifactorial es igual a la cantidad de niveles [4]. Para este experimento serán: cantidad de diésel regulado y cantidad de diésel no regulado.

\subsection{Hipótesis de investigación}

$\mathrm{H}_{0}$ : la regulación de la cantidad de diésel no influye en la calidad de las tejas de arcilla.

$\mathrm{H}_{1}$ : la regulación de la cantidad de diésel influye en la calidad de las tejas.

\subsection{Recolección de datos}

En la recolección de los datos para el análisis, se tomaron nueve muestras de 608 tejas cada una, tres muestras diarias en tres turnos distintos (diurno, vespertino y nocturno) durante tres días de producción. El análisis de las muestras se realizó para las dos condiciones: proceso sin y con regulación de la cantidad de diésel aplicado en la teja; en cada condición señalada se inspeccionaron un total de 5,472 tejas. Para regular la cantidad de diésel aplicado, se colocó una válvula (ver figura 2). El total de muestras fue de 5,472 tejas para cada condición.

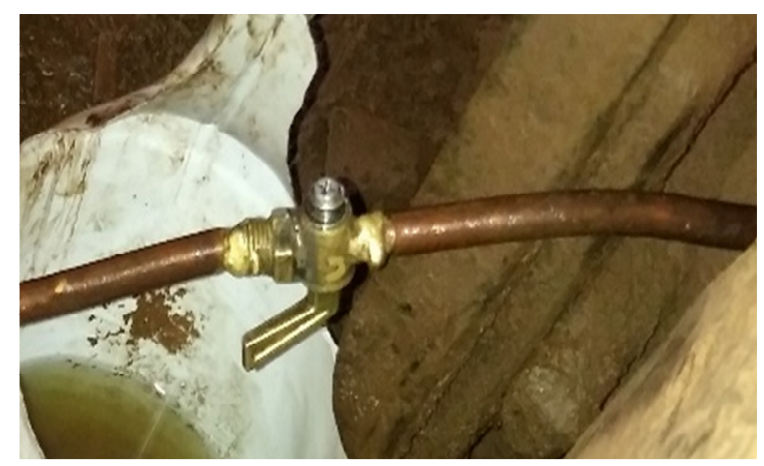

Figura 2. Válvula colocada para regular la cantidad de diésel aplicado.

La selección de la muestra se realizó de forma visual considerando como defectuosas aquellas que no satisfacían las normas de calidad de la empresa, que establecen que la superficie de la teja deberá ser lisa y pareja sin imperfecciones 
visibles a una distancia de 4.5 metros y además estará libre de defectos estructurales [5], (ver figura 3).

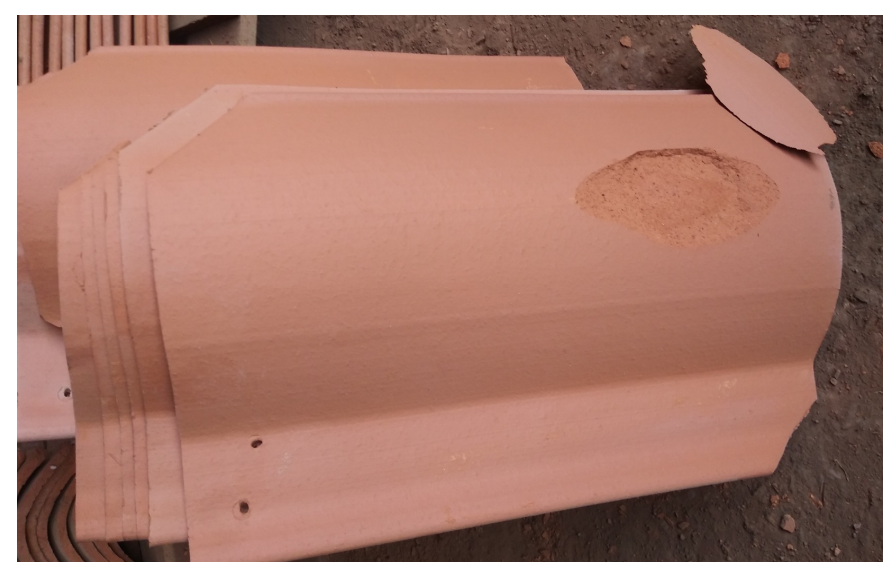

Figura 3. Teja defectuosa.

\section{Análisis de los resultados}

\subsection{Análisis descriptivo}

De acuerdo a los datos recolectados (ver tabla 1), se aprecia una media porcentual de 30.24 tejas defectuosas en cuanto al diésel no regulado y de $4.73 \%$ para el diésel regulado; una variación de 25.51 puntos porcentuales.

Tabla 1. Porcentaje de tejas defectuosas

\begin{tabular}{|c|c|c|}
\hline \multicolumn{3}{|c|}{ Tabla 1. Porcentaje de tejas defectuosas } \\
$\begin{array}{c}\text { Diésel no } \\
\text { regulado }\end{array}$ & $\begin{array}{c}\text { Diésel } \\
\text { regulado }\end{array}$ \\
\hline $\mathbf{1}$ & 33.9 & 1 \\
\hline $\mathbf{2}$ & 53.5 & 2.6 \\
\hline $\mathbf{3}$ & 40.2 & 3.3 \\
\hline $\mathbf{4}$ & 21 & 3 \\
\hline $\mathbf{5}$ & 21.8 & 1.3 \\
\hline $\mathbf{6}$ & 28.2 & 4.5 \\
\hline $\mathbf{7}$ & 16.2 & 19 \\
\hline $\mathbf{8}$ & 23.1 & 2.2 \\
\hline $\mathbf{9}$ & 34.3 & 5.5 \\
\hline
\end{tabular}

Fuente: Los autores.

El cambio en el procedimiento revela una disminución en la variabilidad de 11.61 a 5.55 (6.06 puntos porcentuales), lo cual es significativo si se espera reducir el volumen de tejas defectuosas y evitar altos costos de producción en el proceso de fabricación. Se observa igual comportamiento en los valores de rango -nivel mínimo y máximo-, marcando una disminución de 19 puntos porcentuales (un valor de 37 en el diésel no regulado a un valor de 18 con el método de diésel regulado). Ver tabla 2.

\begin{tabular}{|c|c|c|c|c|}
\hline & Mínimo & Máximo & Media & $\begin{array}{c}\text { Desviación } \\
\text { Estándar }\end{array}$ \\
\hline $\begin{array}{c}\text { Diésel no } \\
\text { regulado }\end{array}$ & 16.20 & 53.50 & 30.24 & 11.61 \\
\hline $\begin{array}{c}\text { Diésel } \\
\text { regulado }\end{array}$ & 1.00 & 19.00 & 4.73 & 5.55 \\
\hline
\end{tabular}

Fuente: Datos obtenidos por los autores.

\subsection{Análisis de varianza}

En la tabla 3 se muestran los resultados del análisis de varianza, para el cual se tomaron los porcentajes de las muestras recolectadas y determinar si existe diferencia en las medias poblacionales de los tratamientos. Se observa el valor crítico de F (Fisher crítica) con un valor de 4.49 a un nivel de confianza del $95 \%$, grados de libertad $(1,16)$, es menor a la Fisher calculada que presenta un valor de 32.36. Esto permite inferir que de acuerdo a los datos recolectados, se rechaza la hipótesis nula y se comprueba de que la regulación en la cantidad de diésel durante el proceso de producción de la teja, sí influye en su calidad.

Tabla 3. Resultados del análisis de varianza

\begin{tabular}{|c|c|c|c|c|c|}
\hline $\begin{array}{c}\text { Origen de } \\
\text { las } \\
\text { variaciones }\end{array}$ & $\begin{array}{c}\text { Suma de } \\
\text { cuadrados }\end{array}$ & $\begin{array}{c}\text { Grados } \\
\text { de } \\
\text { libertad }\end{array}$ & $\begin{array}{c}\text { Promedio } \\
\text { de los } \\
\text { cuadrados }\end{array}$ & F & $\begin{array}{c}\text { Valor } \\
\text { crítico } \\
\text { para F }\end{array}$ \\
\hline Entre grupos & 2928.68 & 1 & 2928.68 & 35.36 & 4.49 \\
\hline $\begin{array}{c}\text { Dentro de los } \\
\text { grupos }\end{array}$ & 1325.26 & 16 & 82.83 & & \\
\hline Total & 4253.94 & 17 & & & \\
\hline
\end{tabular}

Fuente: Datos obtenidos por los autores.

\subsection{Validación de los supuestos}

Se realizó una prueba de Levene para comprobar la homogeneidad de la varianza del porcentaje de tejas defectuosas, a un nivel de significancia de $\infty=5 \%$, los resultados obtenidos se muestran en la tabla 4 , donde $\mathrm{P}>\infty$, por lo que se confirma que existe homogeneidad en la varianza, (ver figura 4).

Tabla 4. Resultados prueba de Levene

\begin{tabular}{|l|l|}
\hline Estadística de Prueba & 4.34 \\
\hline Valor P & 0.054 \\
\hline \multicolumn{2}{|l|}{ Fuente: Datos obtenidos por los autores } \\
\hline
\end{tabular}

\section{Eficiencia productiva}

La tabla 5 muestra la eficiencia productiva y el margen de ganancia bruta asociado a la regulación de la cantidad de diésel empleado, dentro del proceso productivo de la teja. 
Madrid (et al): Efecto de la cantidad de diésel en la calidad de las tejas

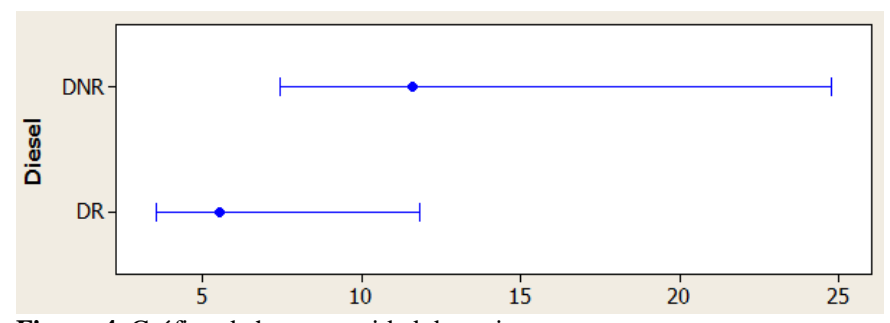

Figura 4. Gráfico de homogeneidad de varianza.

Fuente: Elaborado por los autores.

$\mathrm{El}$ análisis revela un incremento en la eficiencia productiva del 58\%, lográndose un beneficio de $\$ 832.50$ en la utilidad bruta, asumiendo que todas las tejas aceptables son vendidas.

Tabla 5. Eficiencia productiva y margen de ganancia bruta

\begin{tabular}{|c|c|c|}
\hline & Actual & Propuesto \\
\hline Unidades producidas diarias & 1824 & 1824 \\
\hline Unidades defectuosas diarias & 597 & 42 \\
\hline Unidades aceptables diarias & 1227 & 1782 \\
\hline Eficiencia (\%) & 62.27 & 97.70 \\
\hline Incremento de eficiencia (\%) & \multicolumn{2}{|c|}{58} \\
\hline Precio unitario $(\$)$ & \multicolumn{2}{|c|}{1.50} \\
\hline Costo variable (\$) & \multicolumn{2}{|c|}{1.14} \\
\hline \multicolumn{3}{|c|}{ Margen de ganancia bruta } \\
\hline Ingreso por venta $(\$)$ & 1840.50 & 2673.00 \\
\hline Costo variable $(\$)$ & 2079.36 & 2079.36 \\
\hline Utilidad bruta $(\$)$ & $(238.86)$ & 593.64 \\
\hline $\begin{array}{l}\text { Incremento en utilidad bruta } \\
\text { (\$) }\end{array}$ & \multicolumn{2}{|c|}{832.50} \\
\hline
\end{tabular}

Fuentes: Datos recopilado por los autores

\section{Conclusiones}

Los resultados del análisis en esta investigación permiten afirmar que regular la cantidad de diésel durante el proceso de fabricación de las tejas de arcilla, reduce significativamente la cantidad de tejas defectuosas, lo que es un indicador de que sí afecta su calidad. Una posible explicación es que el diésel forma una capa sobre el material estudiado causando que durante el proceso de secado el agua no logre evaporarse por completo y al momento en que se introduzca al horno el agua atrapada por la capa de diésel, tienda a evaporarse más rápido y a medida que se eleva la temperatura, aumenta la presión hasta llegar al punto en que logra salir, provocando defectos estructurales.

\section{AGRADECIMIENTO}

Extendemos nuestro más sincero agradecimiento a la empresa Arcillas S.A. en especial al Ingeniero Benito Suarez por abrirnos las puertas de su empresa y brindarnos la oportunidad de realizar el experimento, igualmente a la ingeniera Maritza de Moreno por su orientación en el cálculo de las muestras que fueron tomadas. A la ingeniera Celinda Pérez De León, por su acertada colaboración en la revisión y redacción final del documento.

\section{REFERENCIAS}

[1] R. Barra, Círculos de Calidad en Operaciones, México: McGRAW-HILL, 1985.

[2] D. C. Montgomery, Control estadístico de la calidad, México: Grupo Editorial Iberoamérica S.A. de C.V., 1991.

[3] D. C. Montgomery, Diseño y Análisis de experimento, México: Grupo Editorial Iberoamérica, S.A de C.V, 1991.

[4] H. G. Pulido, Análisis y Diseño de Experimento, México: McGRAW-HILL, 2008.

[5] A. S.A., «Especificación de los Productos,» Chitré, Panamá. 